\title{
Inhibition of the Hedgehog signaling pathway suppresses cell proliferation by regulating the Gli2/miR-124/AURKA axis in human glioma cells
}

\author{
LIYAO XU ${ }^{1}$, HUA LIU $^{1}$, ZHANGMING YAN ${ }^{2}$, ZHIRONG SUN ${ }^{2}$, SHIWEN LUO $^{1}$ and QUQIN LU ${ }^{3}$ \\ ${ }^{1}$ Center for Experimental Medicine, The First Affiliated Hospital of Nanchang University, Nanchang, \\ Jiangxi 330006; ${ }^{2}$ MOE Key Laboratory of Bioinformatics, School of Life Science, Tsinghua University, \\ Beijing 100084; ${ }^{3}$ Department of Biostatistics and Epidemiology, School of Public Health, \\ Nanchang University, Nanchang, Jiangxi 330006, P.R. China
}

Received February 6, 2017; Accepted March 22, 2017

DOI: 10.3892/ijo.2017.3946

\begin{abstract}
Multiple lines of evidence indicate that aberrant activation of Hedgehog (Hh) signaling plays an important role in tumorigenesis in human glioma. However, the underlying molecular mechanism and crucial downstream targets of glioma-associated oncogene (Gli), a primary transcriptional regulator of Hh signaling, are not fully understood. Here, we report the identification of miR-124 as a novel downstream target of the transcriptional factor Gli2, which is important for proliferation and tumor growth in human glioma cells. Blockade of Hh signaling leads to a remarkable increase in miR-124 expression in glioma cells, whereas overexpression of Gli2 suppresses miR-124 expression by increasing the direct binding of Gli2 to the upstream region of the transcriptional start site for miR-124. Furthermore, we found that miR-124 potentially interacts with the 3'-UTR region of AURKA. Overexpression of miR-124 significantly decreased the expression of AURKA in glioma cells. In contrast, the loss of miR-124 led to the increased expression of AURKA mRNA and protein. In addition, cell proliferation and colony formation ability were significantly decreased following Gli2 knockdown in human glioma cells, while transfection with a miR-124 inhibitor rescued the proliferative ability of cells. These results demonstrate that miR-124 is an important downstream target gene of Hh signaling, and the Gli2/miR-124/ AURKA axis is essential for the proliferation and growth of human glioma cells.
\end{abstract}

Correspondence to: Dr Quqin Lu, Department of Biostatistics and Epidemiology, School of Public Health, Nanchang University, Jiangxi 330006, P.R. China

E-mail: quqinlu@ncu.edu.cn

Key words: Hedgehog signaling pathway, transcription factor Gli, miR-124, AURKA, cell proliferation, human glioma

\section{Introduction}

Glioma is one of the most common and aggressive human malignancies worldwide (1). The poor prognosis of glioma is largely due to the deregulation of intercellular signaling pathways, including Notch and retinoic acid pathways, and the Hedgehog $(\mathrm{Hh})$ signaling pathway, which may promote glioma progression by conferring cell proliferation and survival advantages through several mechanisms (2).

Hh signaling transduction is initiated by the binding of $\mathrm{Hh}$ proteins (sonic Hh, Shh; Indian Hh, Ihh; and Desert $\mathrm{Hh}, \mathrm{Dhh})$ to the 12-pass transmembrane protein Patched $(\mathrm{PTCH})$, which abrogates the repressive activity of $\mathrm{PTCH}$, allowing the 7-pass transmembrane protein Smoothened (Smo) to transduce the signal to the nucleus. Specifically, Smo promotes nuclear translocation of the 5-zinc-finger transcription factors glioma-associated oncogenes (Glis) and subsequently activates target gene transcription $(3,4)$. There are three members in the Gli family, Gli1, Gli2, and Gli3. Gli1 and Gli2 primarily act as transcriptional activators, whereas Gli3 acts as a transcriptional repressor in the Hh signaling pathway (5). Gene targeting studies in mice have demonstrated that Gli2 and Gli3 are the primary mediators of Hh signaling and are essential for embryogenesis. Loss of Gli2 is embryonically lethal, whereas Gli1 is dispensable for animal development $(6,7)$.

In humans, the Hh signaling pathway is critical for embryonic development and adult homeostasis, and Hh signaling activity normally ceases after embryogenesis. However, aberrant activation of the Hh signaling cascade has been shown to be associated with oncogenesis and maintenance of the malignant phenotype in multiple types of human cancers $(8,9)$. Increasing evidence shows that excessive activating mutations in the Smo gene, loss of function mutations in the PTCH gene, or amplification of Glis cause the majority of human cancers (10). RNA analysis of clinical samples found upregulated expression of Gli1 and PTCH1 in glioma tissues (11), and vismodegib treatment reduced Gli1 expression concomitant with the induction of apoptosis and cell cycle arrest (12). Blockade of Hh signaling 
by cyclopamine (13), Gli-ANTagonist 61 (GANT61) (14) or Smo shRNA (15) inhibits cell proliferation and suppresses tumor formation. These observations indicate that deregulated Hh signaling is correlated with rapid growth of human glioma cells. Nevertheless, the mechanisms by which Hh signaling promotes tumor growth need to be further elucidated.

MicroRNAs (miRNAs) are a class of short, singlestranded endogenous non-coding RNAs (approximately 20-22 nucleotides in length) that post-transcriptionally control gene expression via either translational repression and/or mRNA degradation in multicellular eukaryotes (16). Computational and biological analyses estimate that approximately $30 \%$ of all genes and the majority of genetic pathways are subject to regulation by multiple miRNAs (17). By targeting multiple transcripts, miRNAs play important roles in a wide array of biological processes, including development, differentiation, cell proliferation, apoptosis, and metabolism.

It is noteworthy that as a regulatory element, miRNA itself often acts as downstream effector of transcription factors including p53, HIF-1, and c-myc, which have been verified to regulate the expression of several miRNAs (18). Although the diversity and abundance of miRNAs seem to be regulated by several transcription factors and mediate gene expression in any given cancer type, to our knowledge there has been no definitive description of miRNAs whose expression is regulated by $\mathrm{Hh}$ signaling in a transcriptional manner. In addition, the functional consequences of such regulation are ambiguous.

In this study, we performed a set of experiments to elucidate the molecular mechanisms by which the Hh signaling pathway regulates cancer cell proliferation and tumor growth. Our findings indicate that inhibition of Hh signaling suppresses cell proliferation, at least in part, via the Gli2/miR-124/AURKA axis in human glioma cells.

\section{Materials and methods}

Reagents, antibodies and constructs. Primary antibodies were purchased from Millipore (GAPDH, mAb374) and Cell Signaling Technology (Aurka, 4718). A PrimeScript ${ }^{\mathrm{TM}}$ RT reagent kit with gDNA Eraser, SYBR ${ }^{\circledR}$ Premix Ex Taq ${ }^{\mathrm{TM}}$ II and a One Step PrimeScript miRNA cDNA Synthesis kit were purchased from Takara (Tokyo, Japan). The miR-124 inhibitor and control oligonucleotides were purchased from Ribobio (Guangzhou, China) and prepared as $50 \mu \mathrm{M}$ stock solutions using RNase-free $\mathrm{H}_{2} \mathrm{O}$. Lipofectamine 2000 was obtained from Invitrogen Life Technologies (Carlsbad, CA, USA). The working concentrations for small molecular inhibitors and chemicals included GANT61 (20 $\mu \mathrm{M}$, G9048, Sigma) and DMSO (0231, Amresco), which was used as the solvent for the inhibitors and as the vehicle control. Human AURKA (NM_000689) was subcloned between EcoRI and XhoI sites in pcDNA-Flag3.0 (BD Biosciences Clontech, Palo Alto, CA, USA) in-frame downstream of the Flag epitope. shRNA plasmids that separately suppressed the expression of Gli1 (NM_005269.2) and Gli2 (NM_005270) were generated using a BLOCK-iT ${ }^{\mathrm{TM}}$ Pol II miR RNAi Expression Vector kit (K4936-00, Invitrogen Life Technologies). The oligonucleotide sequences for the shRNA constructs are listed in Table I. The authenticity of all constructs was verified by DNA sequencing.

Cell culture and transfection. Human glioma cell lines (H4 and U87) and a human embryonic kidney cell line (HEK293T) were purchased from the American Type Culture Collection (ATCC, Manassas, VA, USA) and cultured in DMEM containing $10 \%$ fetal bovine serum (Gibco, Carlsbad, CA, USA), $50 \mathrm{mg} / \mathrm{ml}$ penicillin $(100 \mathrm{U} / \mathrm{ml})$ and streptomycin $(100 \mu \mathrm{g} / \mathrm{ml})$ at $37^{\circ} \mathrm{C}$ in a humidified atmosphere of $5 \% \mathrm{CO}_{2}$ in air. All cell lines used tested negative for mycoplasma and used in less than 2 months when the experiments were performed. Transient transfection was performed with Lipofectamine 2000 (Invitrogen Life Technologies) according to the manufacturer's instructions.

Gene expression profiling by microarray analysis and real-time PCR. Total RNA, including miRNA, was isolated using TRIzol (Ambion, Austin, TX, USA). The integrity of the total RNA was analyzed by gel electrophoresis. Then, $200 \mathrm{ng}$ of the isolated total RNA was labeled using an Illumina Total Prep-96 RNA Amplification kit (PN:4393543, Ambion Life Technologies, Grand Island, NY, USA), and $750 \mathrm{ng}$ of cRNA was generated and hybridized into a Human HT-12 V4 BeadChip. Then, the BeadChip was washed and stained as per the Illumina protocol and scanned on an iScan (Illumina, San Diego, CA, USA). Data analysis was performed with Genespring GX 12.0 Software (Agilent Technology, Inc., Santa Clara, CA, USA). Raw data were filtered by percentile (lower cut-off: 20). An unpaired t-test was used to identify significant $(\mathrm{P}<0.05)$ gene expression changes with multiple testing correction (Benjamini-Hochberg) to control the false discovery rate and obtain statistically reliable results.

Real-time PCR analysis of the expression of all mRNA and miRNA was analyzed using a SYBR Green kit (Takara) according to the manufacturer's instructions. Briefly, for the detection of mRNA, $1 \mu \mathrm{g}$ of total RNA was used to generate cDNA via reverse transcription using a PrimeScript RT reagent kit with gDNA Eraser. Then, PCR was performed using a SYBR Premix Ex Taq II. For the detection and estimation of miRNAs, we employed a real-time PCR method that involves the formation of miRNA-specific cDNA from total RNA (10 ng) using a specific primer. A diluted reverse transcription product, which is a miRNA-specific cDNA was used for each real-time PCR reaction. The primers for PCR are shown in Table II. All experiments were performed using the ABI StepOnePlus ${ }^{\mathrm{TM}}$ Real-Time qPCR System (Applied Biosystems Inc., Carlsbad, CA, USA), miRNA and mRNA values were normalized to two endogenous controls, U6 and GAPDH, respectively. The $2^{-\Delta \Delta C T}$ method was used to calculate the expression ratios.

Bioinformatic analysis. Identification of transcription factor binding sites in the pre-miR-124 promoter was performed using Cisgenome 2.0 software to identify putative transcription factors that could potentially bind and regulate the expression of miR-124. The motif resembling the known Gli binding site was CTGGGTGGTC (11). A $10 \mathrm{~kb}$ region in the promoter of pri-miR-124 was used for the transcription factor analysis. The public databases TargetScan, PicTar, and 
Table I. Interference sequences.

\begin{tabular}{lll}
\hline Genes & Target site & Target sequence \\
\hline sh-Gli1-720 & 720 to 740 & 5'-TTCATACACAGATTCAGGCTC-3' \\
sh-Gli1-1863 & 1863 to 1883 & 5'-TTCATACACAGATTCAGGCTC-3' \\
sh-Gli1-2255 & 2255 to 2275 & 5'-AAGACCTATCCGATCCAGCGG-3' \\
sh-Gli2-233 & 233 to 253 & 5'-AATGGTACCTTCCTTCCTGGT-3' \\
sh-Gli2-1127 & 1127 to 1147 & 5'-TGGCCTGAAACGATGTCATC-3' \\
sh-Gli2-2058 & 2058 to 2078 & 5'-TGTGAATGGCGACAGGGTTGA-3'
\end{tabular}

Table II. Primer sequences for real-time PCR.

\begin{tabular}{lll}
\hline Genes & \multicolumn{1}{c}{ Forward primer (5' to 3') } & \multicolumn{1}{c}{ Reverse primer (5' to 3') } \\
\hline Gli1 & 5'-TCCTACCAGAGTCCCAAGTT-3' & 5'-CCCTATGTGAAGCCCTATTT-3' \\
Gli2 & 5'-CTGTGGGTTAGGGATGGACTG-3' & 5'-GTAAAGTGGGTGGACGTTGCA-3' \\
AURKA & 5'-GGAATATGCACCACTTGGAACA-3' & 5'-TAAGACAGGGCATTTGCCAAT-3' \\
GAPDH & 5'-GAAGGTGAAGGTCGGAGTC-3' & 5'-GAAGATGGTGATGGGATTTC-3' \\
miR-301b & 5'-GCAGTGCAATGATATTGTCAAAGC-3' & Uni-miR PCR Primer \\
miR-302d & 5'-GCTAAGTGCTTCCATGTTTGAGTGT-3' & Uni-miR PCR Primer \\
miR-519a & 5'-GCAAAGTGCATCCTTTTAGAGTGT-3' & Uni-miR PCR Primer \\
miR-335 & 5'-GCTCAAGAGCAATAACGAAAAATGT-3' & Uni-miR PCR Primer \\
miR-122 & 5'-GTGGAGTGTGACAATGGTGTTTG-3' & Uni-miR PCR Primer \\
\hline
\end{tabular}

Miranda were used to identify putative miRNA seed matching sequences in the 3'-UTR of AURKA.

Chromatin immunoprecipitation. A chromatin immunoprecipitation (CHIP) analysis was performed to detect the occupation of the Gli2 transcription factor on the putative regions of miR-124. In brief, $\mathrm{H} 4$ cells were cultured in a $10-\mathrm{cm}$ dish until reaching approximately $90 \%$ confluence. After discarding the original medium, H4 cells were crosslinked with $5 \mathrm{ml}$ of PBS containing $1 \%$ formaldehyde at room temperature for $10 \mathrm{~min}$ with gentle shaking. Then, DNA was sonicated into a range of 200-1000 base pairs in size using a Bioruptor Sonicator (Diagenode) for five cycles of $3 \mathrm{sec}$ on $/ 3 \mathrm{sec}$ off. The extracts were pre-cleared in BSA-blocked protein A beads and incubated with anti-Gli2 or IgG control overnight at $4^{\circ} \mathrm{C}$. After being washed, DNA was eluted and reverse cross-linked overnight at $65^{\circ} \mathrm{C}$ and then purified and amplified by PCR. The primers for PCR are shown in Table III.

Luciferase assay. The wild-type (WT) AURKA-3'-UTR was amplified by PCR from human cDNA using the primers (forward) 5'-CAA GCT TCA CAT CAG GTG GAT GGA GAG AC-3' and (reverse) 5'-GAG CTC GGC AGG GGA AAG CTG TAG GAA T-3'. The mutant-type (Mut) AURKA-3' UTR was amplified using the primers (forward) 5'-CAA GCT TCA CAT CAG GTG GAT GGA GAG AC-3' and (reverse) 5'-GAG CTC GGC AGG GGT ATG GTC TAG GAA T-3'. Then, the cDNA fragments were inserted into a pGL3 Vector using the SacI and HindIII sites.
HEK293T cells were cultured in 24-well plates and co-transfected with pGL3.0 vectors containing either the WT or mutated AURKA-3'-UTR vectors and miR-124 expression plasmid or control plasmid using Lipofectamine 2000. After $48 \mathrm{~h}$, cells were lysed, and luciferase assays were performed using a dual luciferase reporter assay kit (Promega, Madison, WI, USA). Renilla luciferase activity was used to normalize the transfection efficiency. Three independent experiments were performed in triplicate.

Western blotting. Cells were washed with chilled PBS and harvested by trypsinization. Then, cells were lysed in lysis buffer at $4^{\circ} \mathrm{C}$ for $30 \mathrm{~min}$ and centrifuged (12,000 rpm, $15 \mathrm{~min}$ at $4^{\circ} \mathrm{C}$ ) to collect the supernatant. Protein concentrations were determined by the BCA method using Pierce ${ }^{\mathrm{TM}}$ BCA Protein Assay kit (Thermo Scientific, Rockford, IL, USA) according to the manufacturer's instructions. Subsequently, the lysates were separated on SDS-PAGE gels and immunoblotted using standard procedures. The primary antibodies used were anti-AURKA (Abcam, 1:1000) and anti-GAPDH (Millipore, 1:2000). Finally, immunostaining was visualized using Kodak X-ray film, which was subsequently scanned with an Epson 1680 scanner. Quantitative analysis was performed on scanned images of blots using ImageJ software.

Cell viability and colony formation assays. Cell viability assays were performed as previously described (19). H4 cells $\left(\sim 5 \times 10^{3}\right.$ per well) were seeded into a 96 -well plate and cultured for $72 \mathrm{~h}$. Then, MTT solution was added and cells were incubated for $4 \mathrm{~h}$. The remaining MTT formazan crystals were 
Table III. Primer sequences for CHIP.

\begin{tabular}{lll}
\hline Genes & \multicolumn{1}{c}{ Forward primer (5' to 3') } & \multicolumn{1}{c}{ Reverse primer (5' to 3') } \\
\hline BS1 & 5'-TACAGAGGGATCTGTTGGGAGT-3' & 5'-TGGCCTTACCTACAAAATGGG-3' \\
BS2 & 5'-AGGCTGGTTTCAAACTCCTG-3' & 5'-TAGTGTCTAGGCTGGGTGC-3' \\
BS3 & 5'-AGGGAAATGATTCCAAGCC-3' & 5'-CTGGGAAGTTCTGAATGTTTG-3' \\
BS4 & 5'-GAACTTCCCAGTCTAAACAGC-3' & 5'-GGCTTAGGGATTGCTACAAC-3' \\
BS5 & 5'-CGCTTCCAACCTCCTCTTG-3' & 5'-GGGCTGGTCTTGAACTCCT-3' \\
BS6 & 5'-GCTGGGAACTGTAGTCTTGC-3' & 5'-GCCACTGGAGGTAGTGATT-3' \\
BS7 & 5'-TTCTTCCCAGCAGAGTCAAG-3' & 5'-TAATACCTCGCAAAGCATGG-3' \\
\hline
\end{tabular}

solubilized in DMSO, and the absorbance was measured at OD490. For the colony formation assays, $\mathrm{H} 4$ cells $\left(\sim 3 \times 10^{3}\right.$ per well) were equivalently plated in 6-well plates in DMEM with $10 \%$ FBS. Then, the cells were cultured, and the medium was changed every 5 days. Cells were cultured for up to 12 days. Then, the cells were washed with PBS, fixed with $4 \%$ paraformaldehyde and stained with $0.1 \%$ crystal violet. Dishes were graphed and positive colonies containing more than 50 cells were counted under a microscope. Colony-formation rates were then calculated.

Statistical analysis. The statistical significance between two groups was calculated by unpaired Student's t-test using SPSS 16.0 software. For experiments involving more than one group for comparison, ANOVA was used with a suitable post hoc test. All data are expressed as the mean \pm SD for experiments performed at least three times. Differences were considered significant at $\mathrm{P}<0.05$ or $\mathrm{P}<0.01$.

\section{Results}

Inhibition of Hh signaling results in miRNAome alteration. To identify miRNAs potentially regulated by Hh signaling, we treated H4 cells with GANT61, a specific inhibitor of Gli1 and Gli2 (14). As expected, GANT61 inhibited the expression of Gli1 and Gli2 (Fig. 1A). Subsequently, we purified total RNA species from $\mathrm{H} 4$ cells that were treated with DMSO (Vehicle) or GANT61 $(20 \mu \mathrm{M}, 48 \mathrm{~h})$ and then labeled miRNAs with fluorescent dyes and hybridized them to an oligonucleotide array representing known miRNAs. As shown in Fig. 1B, upon efficient blockade of the Hh signaling pathway by GANT61, a total of 34 miRNAs were significantly overexpressed or underexpressed (Fig. 1B). To confirm the microarray-based observations described above, we validated several mature miRNAs using real-time PCR with stem-loop primers (Fig. 1C). As expected, several mature miRNAs (i.e., miR-124, miR-302d, miR-519a, miR-335 and miR-122) were induced by GANT61 treatment in the tested cell lines, which was consistent with the microarray data. Together, our results suggest that deregulation of Hh signaling may be involved in the regulation of miRNA generation. Similar to other cancers, there is a characteristic miRNA expression pattern in human glioma cells (20). In particular, miR-124, whose mature sequences are conserved from Caenorhabditis elegans to humans, is one of the most deficient miRNAs in glioma tissue compared with normal brain tissue $(20,21)$. In addition, glioma-associated loss of normal brain-enriched miR-124 enhances stem-like traits and the invasiveness of glioma cells (22). Therefore, we decided to direct our attention toward miR-124, given that miR-124 may have critical roles in human glioma tumorigenesis and progression.

In vertebrates, the Gli family of transcription factors, specifically Gli1 and Gli2, mediates the Hh signaling pathway by regulating the transcription of target genes. Their cooperative roles are vital in Hh signaling, while their specific roles have only been partially defined. To interrogate which one of the Glis influences miR-124 biogenesis, we transfected H4 cells with either a Gli1-shRNA or Gli2-shRNA plasmid. We found that sh-Gli1-2855 and sh-Gli2-228 were more efficient in knocking down endogenous Gli1 and Gli2 expression (Fig. 1D and E). miR-124 was only repressed after cells were transfected with Gli2-shRNA plasmid, while Gli1-shRNA had little effect on miR-124 expression in H4 cells. Similar results were also observed in U87 cells (Fig. 1F). Taken together, these results reveal a previously unrecognized function of Hh signaling in miRNA biogenesis, in which Gli2 negatively regulates the expression of miR-124 in human glioma cells.

Expression of $m i R-124$ is regulated by Gli2. To investigate the molecular mechanism by which Gli2 orchestrates miR-124 expression, we measured the expression level of pri-miR-124 and pre-miR-124 following Gli2 knockdown in H4 cells. As shown in Fig. 2A, pri-miR-124 was upregulated after Gli2 depletion in $\mathrm{H} 4$ cells and U87 cells, suggesting a transcriptional level of regulation. Moreover, an increase in pre-miR-124 was also observed in both $\mathrm{H} 4$ cells and U87 cells when transfected with shRNA-Gli2-228 plasmid (Fig. 2B). Thus, we suspected that Gli2 might regulate the transcription of miR-124 in glioma cells.

Next, in order to further illustrate the mechanism, a search for putative Gli2 binding sites, using Cisgenome 2.0, identified seven putative Gli2-binding DNA elements (BS1: +9789 +9798, BS2: +9255 +9234, BS3: +6235 +6244, BS4: +6025 +6034, BS5: +5538 +5547, BS6: +2935 +2944, and BS2: $+551 \sim+560)$ located upstream of the transcriptional start site of miR-124 (Fig. 2C). Subsequently, we conducted a set of ChIP assays in $\mathrm{H} 4$ cells and determined that Gli2 binds to BS2, but not to any of the other binding sites, in the miR-124 upstream sequence (Fig. 2D). These findings suggest that Gli2 may be at least partially responsible for the miR-124 downregulation observed in glioma cells. 
A

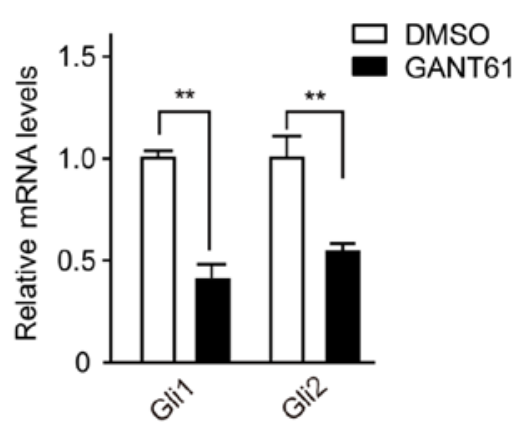

C

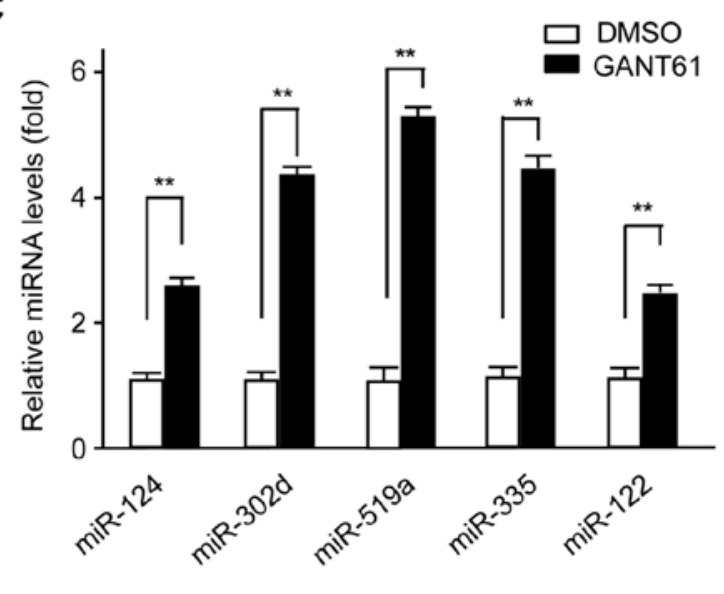

D

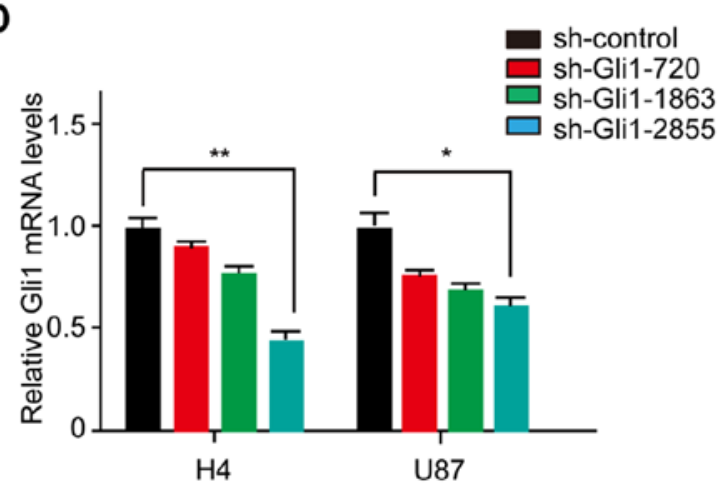

$\mathbf{E}$

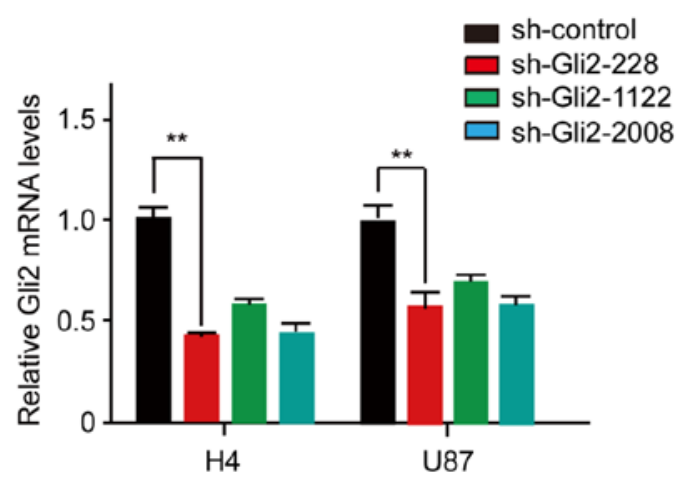

B

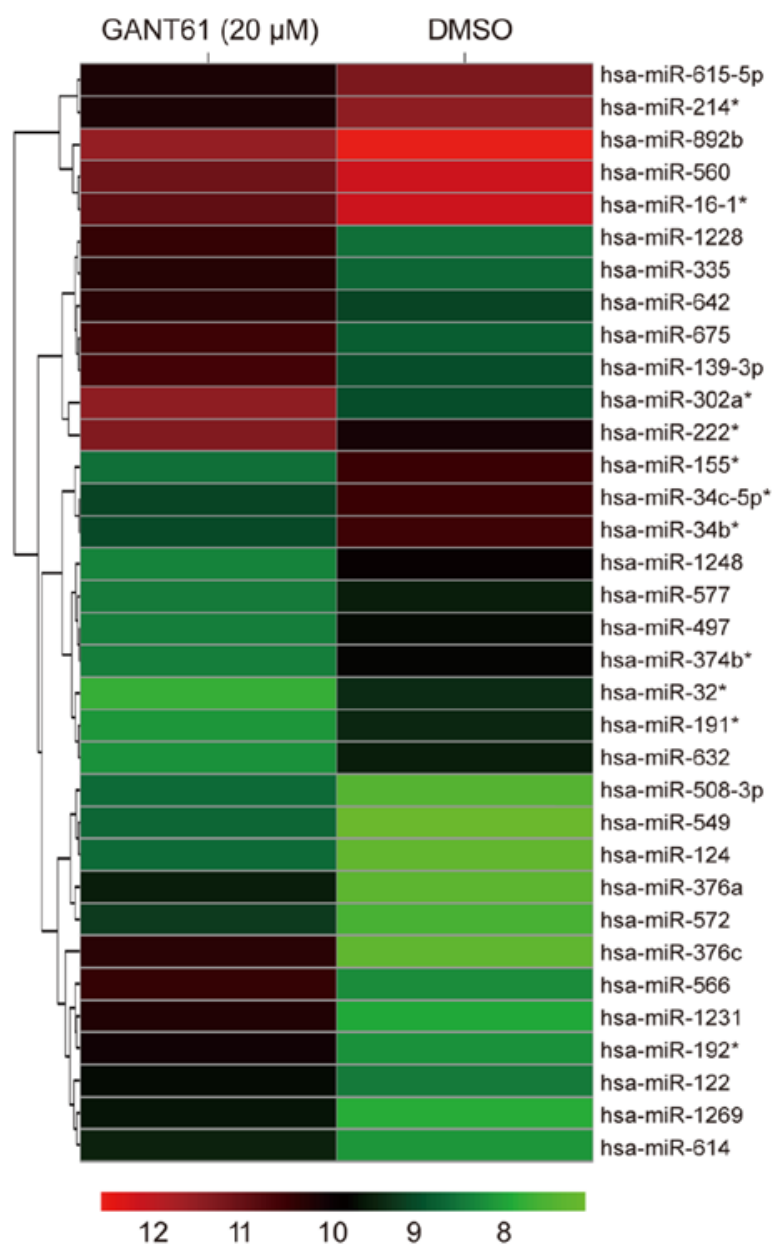

$\mathbf{F}$

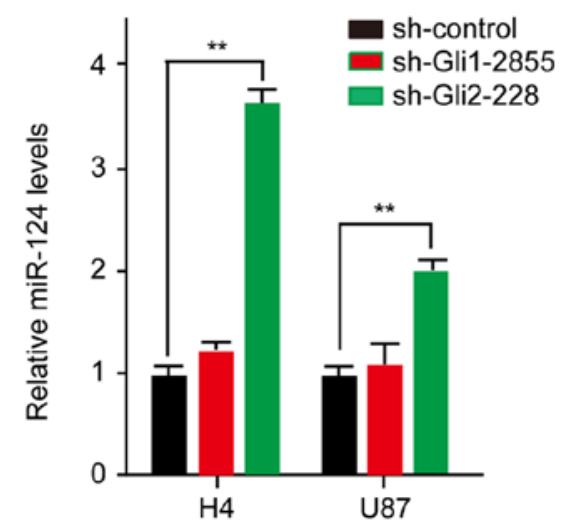

Figure 1. Hedgehog (Hh) signaling is involved in the regulation of miRNA expression. (A) Gli was downregulated in H4 cells by treatment with GANT61 after $48 \mathrm{~h}$. Real-time PCR was performed to analyze Gli mRNA in H4 cells after treatment with DMSO (control) or GANT61 (20 $\mu \mathrm{M})$ for $48 \mathrm{~h}$. (B) Genometric mean-centered, hierarchical cluster heat map form of the microarray data. Microarray analysis of miRNA expression was performed with total RNA extracted from H4 cells treated with DMSO (control) or GANT61 $(20 \mu \mathrm{M})$ for $48 \mathrm{~h}$. The heat map shows the clustering of differentially expressed miRNAs. (C) The expression of miRNAs was detected with real-time PCR after treatment with DMSO (control) or GANT61 (20 $\mu \mathrm{M})$ for $48 \mathrm{~h}$. U6 RNA was used as a loading control. Data are shown as the means \pm SD. (D) The interference efficiency of Gli1 shRNA detected in glioma cells (U87 cells and H4 cells). The expression of Gli1 was quantified with real-time PCR. (E) The interference efficiency of Gli2 shRNA detected in glioma cells (U87 cells and H4 cells). The expression of Gli2 was quantified with real-time PCR. (F) The expression of miR-124 was detected with real-time PCR after transfection with Gli1-miRNA and Gli2-miRNA ( $48 \mathrm{~h}$ ) in $\mathrm{H} 4$ cells and U87 cells. The expression of miR-124 was quantified with real-time PCR. Error bars represent the standard deviation of three independent experiments. ${ }^{*} \mathrm{P}<0.05 ;{ }^{* *} \mathrm{P}<0.01$. 
A

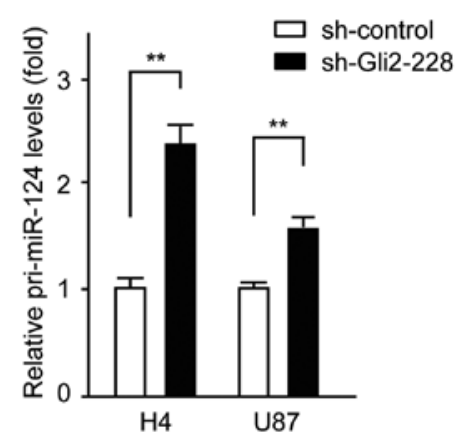

B

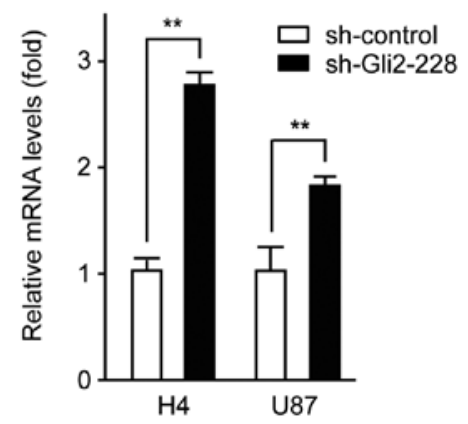

C

miR-124 Upstream Region

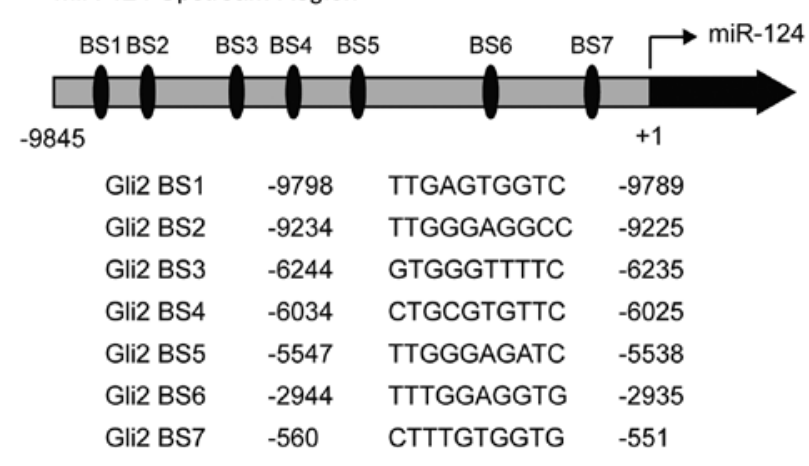

D

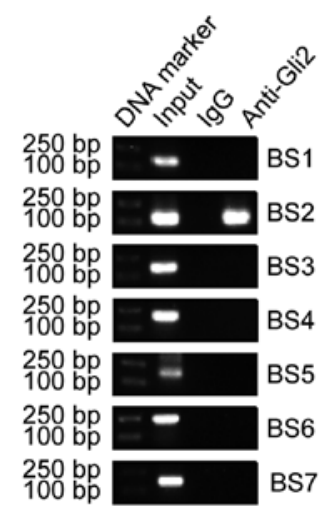

Gli2 Binding Site: GTGGGTGGTC

Figure 2. Gli2 binds directly to the miR-124 genomic locus. (A) H4 cells and U87 cells were transfected with control plasmid or sh-Gli2-228 plasmid and were subjected to real-time PCR analysis to determine pri-miR-124 expression. (B) H4 cells and U87 cells were transfected with either control plasmid or sh-Gli2-228 plasmid (48 h). The expression of pre-miR-124 was quantified by real-time PCR. (C) Schematic diagrams of Gli2 regions indicated the putative Gli2-binding sites in the upstream region of the miR-124 transcription start site. (D) Chromatin was isolated from H4 cells, and ChIP assays were performed with control (IgG) and anti-Gli2 antibodies. Specific primers for each putative binding element were used for PCR analyses (Table III).

AURKA is a direct target of miR-124. To elaborate the functional consequences of the Gli2-mediated inhibition of miR-124 expression, we analyzed the target genes of miR-124. Noteworthy, based on the bioinformatic analysis of potential miR-124 targets (www.miRNA.org), we determined that miR-124 may interact with the 3'-UTR region of AURKA (Fig. 3A). AURKA expression promotes centrosome maturation and separation, which plays multiple roles in cancer development (23). To examine whether miR-124 can regulate AURKA expression, a plasmid construct was used to upregulate the expression of miR-124 in glioma cells. The miR-124 expression level was upregulated in $\mathrm{H} 4$ cells transfected with the miR-124 expression plasmid. Then, we analyzed the expression levels of AURKA mRNA, and the results indicated that AURKA mRNA was decreased by ectopic expression of miR-124 (Fig. 3B and C).

In addition, overexpression of miR-124 significantly decreased the expression of AURKA protein in $\mathrm{H} 4$ cells (Fig. 3D and E). In contrast, the loss of miR-124 in H4 cells transfected with the miR-124-inhibitor led to the increased expression of AURKA mRNA and protein when compared to cells transfected with the control plasmids (Fig. 3F-I). To further confirm that AURKA was directly targeted and regulated by miR-124, luciferase reporter genes with the AURKA 3'-UTR and a mutant counterpart, mutated at the miR-124 binding regions, were co-transfected with the miR-124 expression plasmid or control plasmid into HEK293T cells. The luciferase reporter assay showed that overexpression of miR-124 significantly inhibited the luciferase activity of AURKA with the wild-type 3'-UTR but not with the mutant 3'-UTR (Fig. 3J). These findings demonstrated that AURKA is a direct target gene of miR-124.

Gli2 regulates the expression of AURKA through miR-124. The results described above show that Hh signaling inhibits the expression of miR-124, and miR-124 downregulates the expression level of AURKA (Figs. 2 and 3). In addition, our previous microarray data showed that AURKA was poorly expressed in the GANT61 group compared with the control group in $\mathrm{H} 4$ cells (24). We next investigated if AURKA can be directly regulated by Gli2. It is worth noting that we did not detect any Gli2 occupancy in the analysis of the AURKA binding sites (data not shown), which indicates that AURKA is presumably not a direct transcriptional target of Hh signaling but can be regulated by Hh signaling via miR-124. To validate our hypothesis, we transfected the miR-124 inhibitor into $\mathrm{H} 4$ cells, accompanied by GANT61.

A real-time PCR assay showed that the AURKA mRNA level was downregulated in the GANT61 group, while the miR-124-inhibitor rescued the expression of AURKA mRNA (Fig. 4A). When the expression of miR-124 was prevented by transfection with the miR-124-inhibitor, the inhibitory 
A

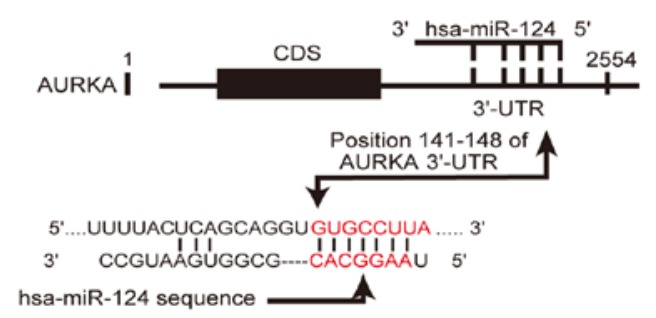

D

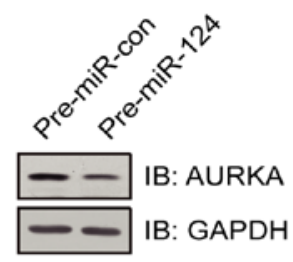

H

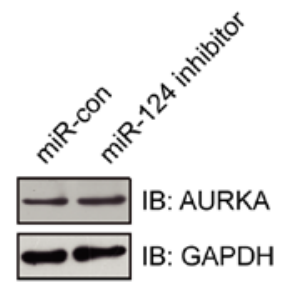

B

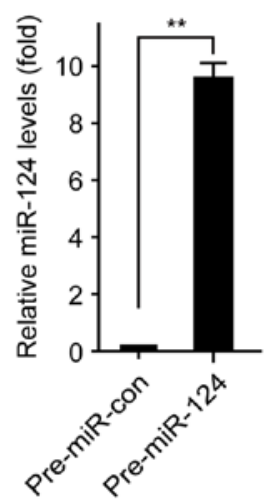

E

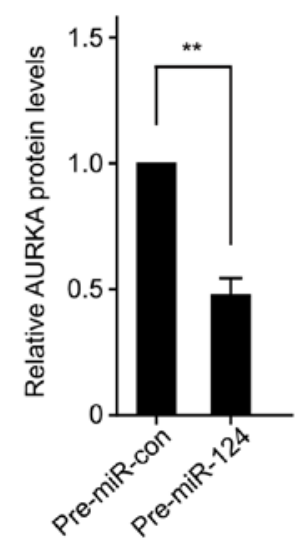

I

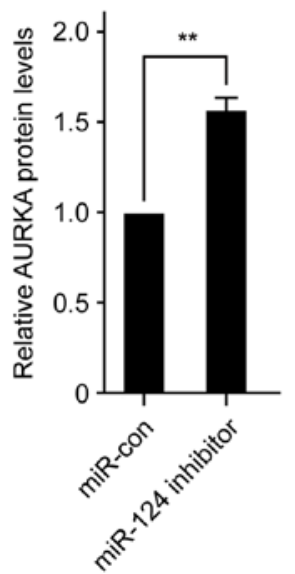

J
C

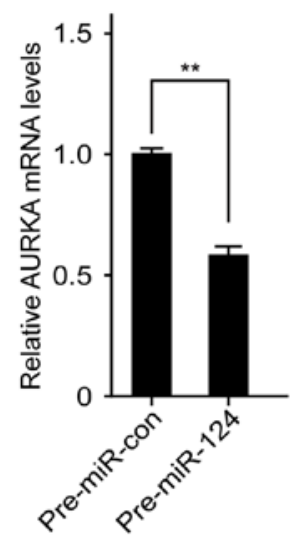

G
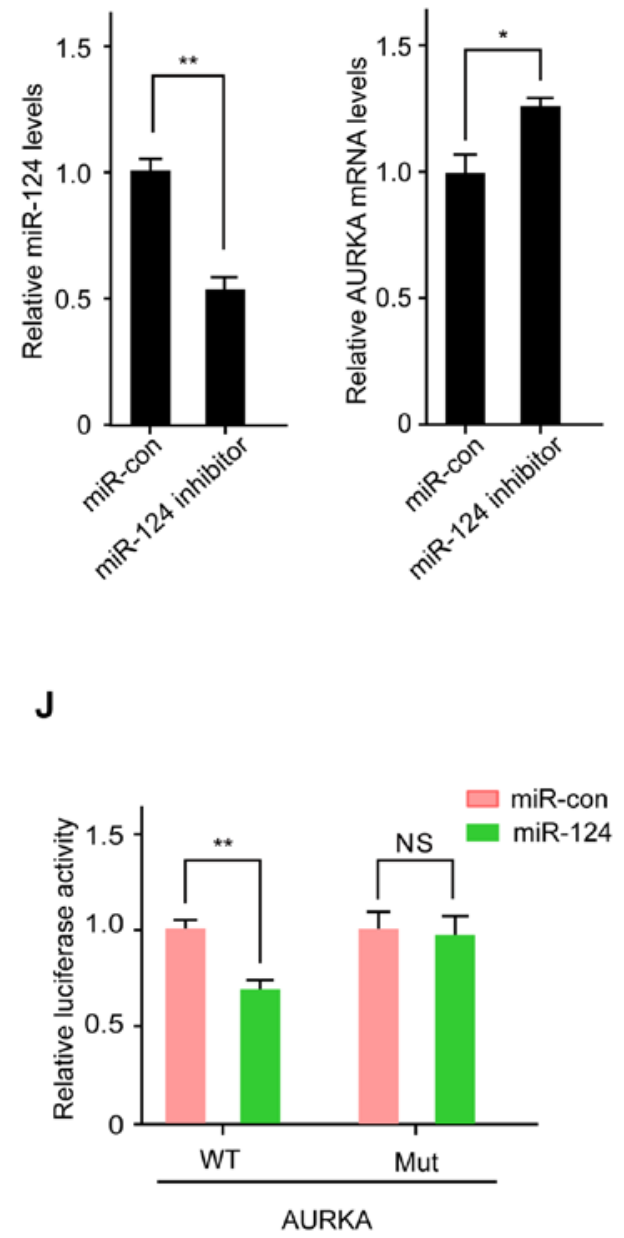

Figure 3. AURKA is a direct target of miR-124. (A) miR-124 sequences and the predicted miR-124 binding sites in the 3'-UTR of human AURKA. (B) Overexpression of miR-124 in H4 cells. H4 cells were transfected with control plasmid or miR-124 expression plasmid for $48 \mathrm{~h}$, and then, miR-124 levels were detected by real-time PCR. (C) miR-124 expression downregulates the mRNA level of AURKA in H4 cells. H4 cells were transfected with control plasmid or miR-124 expression plasmid for $48 \mathrm{~h}$, and then, AURKA mRNA levels were detected with real-time PCR. (D) miR-124 expression downregulates the protein level of AURKA in $\mathrm{H} 4$ cells. Cells were transfected with control plasmid or miR-124 expression plasmid for $48 \mathrm{~h}$, and then, AURKA protein levels were detected by western blotting. (E) Downregulation of the expression of miR-124 in H4 cells. Cells were treated with control oligonucleotides or miR-124 inhibitor for $48 \mathrm{~h}$, and then, miR-124 levels were detected with real-time PCR. (F) Downregulation of the expression of miR-124 in H4 cells. Cells were treated with control oligonucleotides or miR-124 inhibitor for $48 \mathrm{~h}$, and then, AURKA mRNA levels were detected with real-time PCR. (G) Downregulation of the expression of miR-124 in H4 cells. Cells were treated with control oligonucleotides or miR-124 inhibitor for $48 \mathrm{~h}$, and then, AURKA protein levels were detected by western blotting. (H,I,J) The miR-124 binding site in the human AURKA 3'-UTR mediates the repression of luciferase activity in HEK-293T cells. Luciferase reporter constructs containing the wild-type or mutant human AURKA 3'-UTR were fused to the 3'-end of the firefly luciferase gene. Then, the AURKA 3'-UTR luciferase plasmid was transfected into HEK293T cells together with or without the miR-124 expression plasmid. The relative luciferase activity was measured $48 \mathrm{~h}$ after transfection with a dual luciferase assay. The values shown are the means \pm SD for triplicate samples. ${ }^{*} \mathrm{P}<0.05 ;{ }^{* *} \mathrm{P}<0.01 ;$ NS, no significance. 
A

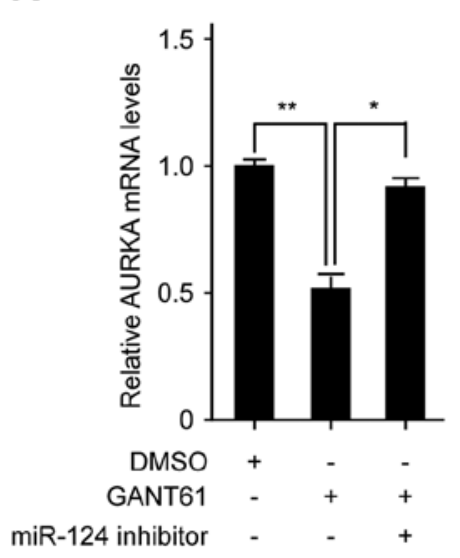

D

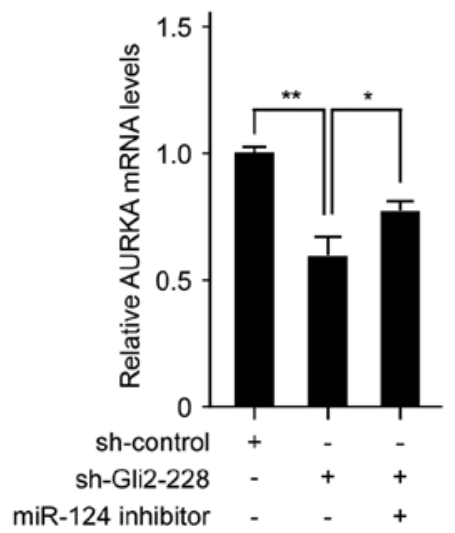

B

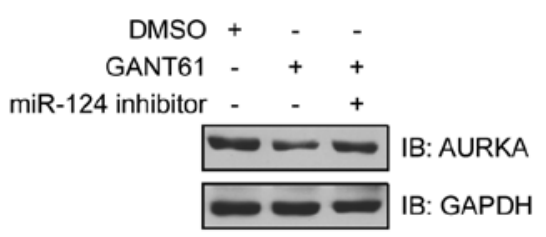

E

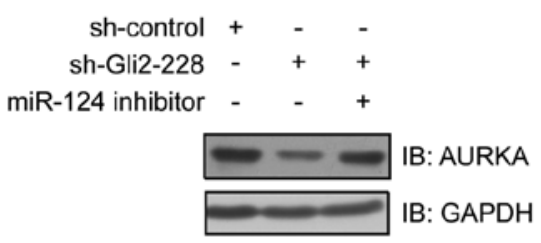

C

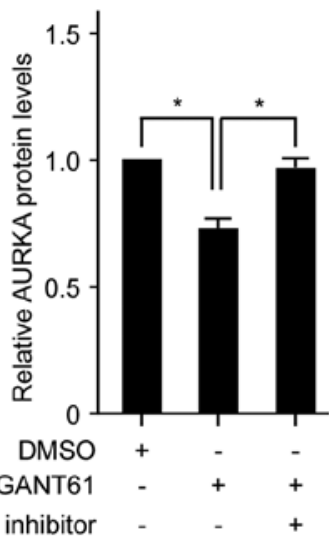

$\mathbf{F}$

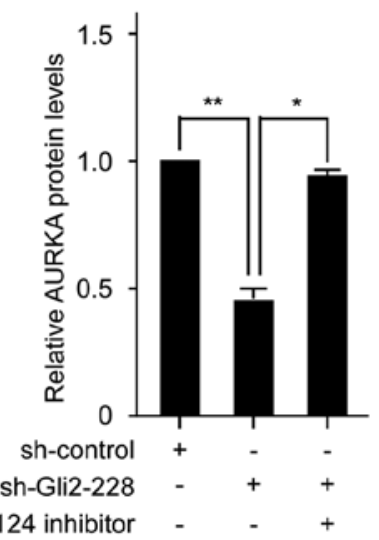

Figure 4. Gli2 influences the expression of AURKA via miR-124. (A) $\mathrm{H} 4$ cells were transfected with miR-124 inhibitor for $12 \mathrm{~h}$ and subsequently treated with GANT61 $(20 \mu \mathrm{M})$ for $48 \mathrm{~h}$. Samples were collected for real-time qPCR to analyze the mRNA expression of AURKA. (B and C) H4 cells were transfected with miR-124 inhibitor for $12 \mathrm{~h}$ and subsequently treated with GANT61 $(20 \mu \mathrm{M})$ for $48 \mathrm{~h}$. AURKA protein was detected by western blotting. (D) H4 cells were transfected with sh-Gli2-228 plasmid and miR-124 plasmid as indicated for $48 \mathrm{~h}$. AURKA mRNA was detected with real-time PCR. (E and F) H4 cells were transfected with sh-Gli2-228 plasmid and miR-124 plasmid as indicated for $48 \mathrm{~h}$. AURKA protein was detected by western blotting. The data shown are the means $\pm \mathrm{SD}$ for triplicate samples. ${ }^{*} \mathrm{P}<0.05 ;{ }^{* *} \mathrm{P}<0.01$.

effect of GANT61 on AURKA protein could be alleviated (Fig. 4B and C). In addition, we inhibited Gli2 in $\mathrm{H} 4$ cells and then knocked down miR-124 expression using the miR-124 inhibitor. We found that after induction of conditional expression of Gli2, a significant reduction in AURKA mRNA was observed in $\mathrm{H} 4$ cells. Of note, knockdown of miR-124 abrogated Gli2-dependent suppression of AURKA mRNA expression (Fig. 4D). Moreover, the expression of AURKA protein was also rescued by co-transfection with the miR-124-inhibitor (Fig. 4E and F). These data indicate that miR-124 acts as a downstream effector of Gli2, and the repression of AURKA through Gli2 inhibition is mediated by miR-124.

Gli2 enhances glioma cell proliferation via the miR-124/AURKA axis. To illustrate the molecular mechanisms by which Hh signaling regulates the proliferation process in glioma cells, we inhibited the expression of Gli2 in $\mathrm{H} 4$ cells. Strikingly, the number of colonies formed by $\mathrm{H} 4$ cells was significantly decreased following Gli2 knockdown, while transfection with the miR-124 inhibitor rescued the proliferative ability of the cells (Fig. 5A and B). To functionally characterize miR-124 in glioma cells, we upregulated miR-124 levels by ectopically expressing miR-124 in H4 cells. Then, colony formation assays were performed to assess the role of miR-124 in cell proliferation. The cells transfected with miR-124 clearly grew slower than in the control group, while co-transfection with the AURKA construct upregulated the number of colonies in $\mathrm{H} 4$ cells (Fig. 5C and D). In addition, an MTT assay further supported the colony formation assay findings (Fig. 5E and F). Collectively, these results suggest that the Gli2/miR-124/AURKA axis can influence the proliferation of human glioma cells.

\section{Discussion}

In this study, we determined that miR-124 acts as a downstream effector of the Hh signaling pathway. Noteworthy, we found that miR-124 potentially interacts with the 3'-UTR region of AURKA. Further experiments showed that the Hh signaling pathway regulated the expression of AURKA through miR-124, and overexpression of miR-124 significantly decreased the expression of AURKA and the proliferation of glioma cells. Our results suggest that the Gli2/miR-124/AURKA axis is essential for the proliferation and growth of human glioma cells.

The Gli transcription factors constitute the final effectors of the Hh signaling pathway, which is frequently hyperactivated 
A

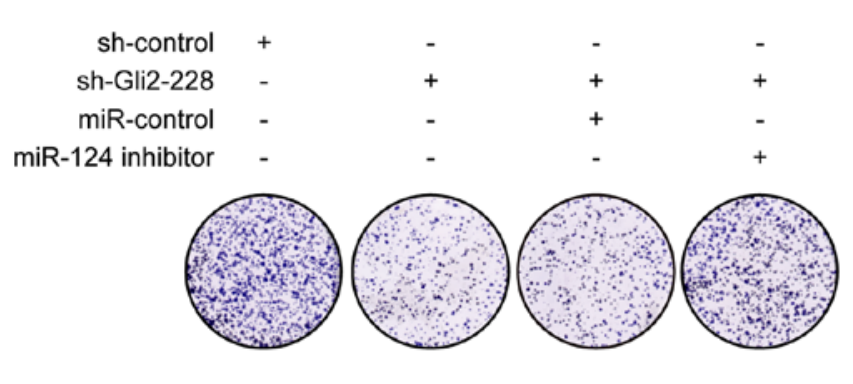

B

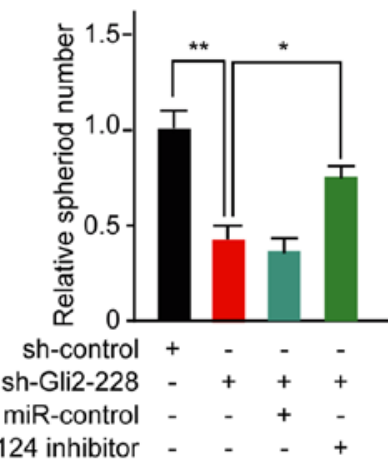

D
C

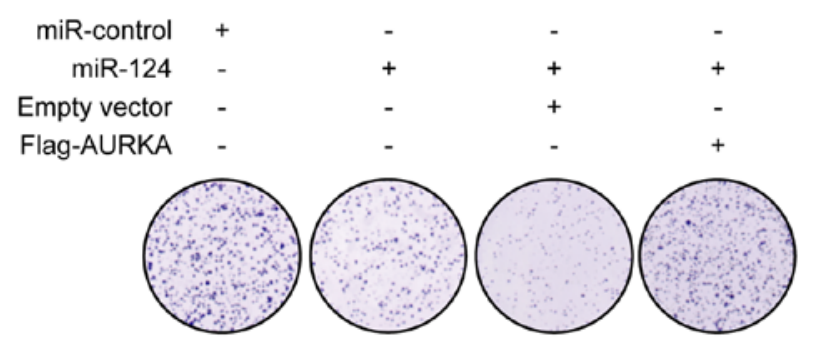

E

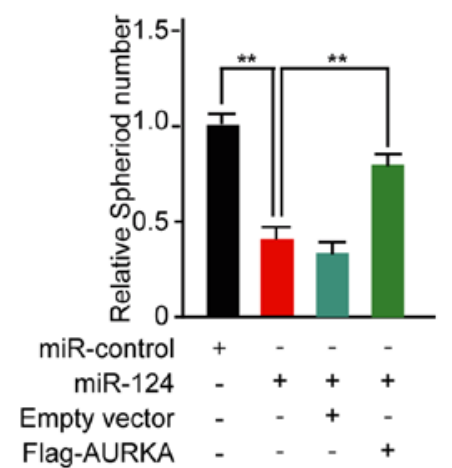

$\mathbf{F}$

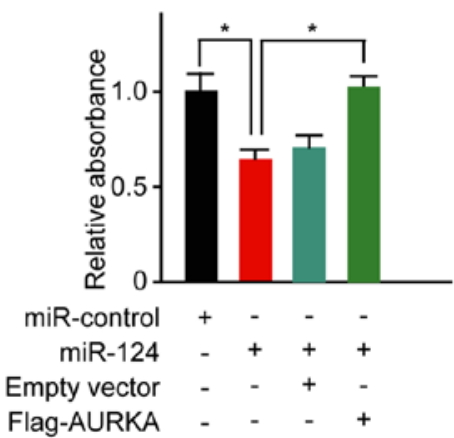

Figure 5. Gli2/miR-124/AURKA mediates the growth of glioma cells. (A and B) Inhibition of miR-124 influences the effect of Gli2 on proliferation. H4 cells were transfected with sh-Gli2-228 plasmid and miR-124 inhibitor as indicated. The proliferation of H4 cells was assessed by a colony formation assay (12 days). The quantitative analysis was performed using ImageJ software. (C and D) Overexpression of AURKA restores the inhibitory effect of miR124 on proliferation. H4 cells were transfected with miR-124 and Flag-AURKA plasmid as indicated, and the proliferation of H4 cells was assessed by a colony formation assay (10 days). The quantitative analysis was performed using ImageJ software. (E) H4 cells transfected with sh-Gli2-228 plasmid were co-transfected with NC or miR-124 inhibitor for $72 \mathrm{~h}$. An MTT assay was performed to determine the proliferation of H4 cells. (F) H4 cells transfected with miR-124 overexpression plasmid were co-transfected with NC or Flag-AURKA plasmid for $72 \mathrm{~h}$. An MTT assay was performed to determine the proliferation of $\mathrm{H} 4$ cells. The bar graph shows the means $\pm \mathrm{SD}, \mathrm{n}=5 ;{ }^{*} \mathrm{P}<0.05 ;{ }^{* *} \mathrm{P}<0.01$.

in human cancers through multiple mechanisms. Hence, targeting Gli may offer a highly effective therapeutic strategy for the treatment of lethal tumors. Currently, there are multiple studies aimed at assessing the efficacy of Gli inhibitors in cancers. However, the downstream mechanisms initiated by Gli are poorly understood, in part because relatively little is known about the multiple specific genes directly regulated by Gli. Before the discovery of noncoding RNAs, searches for transcription factor-targeted genes were focused on protein-coding genes. Intriguingly, it is worth noting that recently several transcription factors have been discovered to regulate the expression of miRNAs.
The central tumor suppressor p53 enhances the transcriptional activity of the miR-34 family by binding to the promoter of miR-34a (25). The proto-oncogene c-myc encodes a transcription factor that regulates cell proliferation and apoptosis, and a CHIP analysis has shown that c-myc binds directly to the locus of a cluster of six miRNAs on human chromosome 13 (26). In addition, interactions between the Hh signaling pathway and miRNA have been recently demonstrated by the discovery that several key components of the Hh signaling pathway are regulated by miRNAs (27-29). For example, in hepatic stellate cells, the expression of Gli2 was markedly inhibited by miR-200a (29). In addition, miR-324-5p resulted in a reduction 
of Gli1 in MB cells (27). Moreover, miR-326 acts as a negative modulator of the $\mathrm{Hh}$ signaling pathway by directly targeting Gli2 (30). These findings strongly suggest that regulation of components of the Hh signaling pathway, such as Glis, by miRNAs contributes to the functions of Hh signaling.

In this study, we demonstrated that Gli2 can directly modulate the expression of miR-124 by binding to one binding site in the upstream region of the transcriptional start site, thereby fine tuning the function of miR-124. Notably, most studies have focused attention on protein-coding genes that can be regulated by Hh signaling. Our findings suggest that, in addition to many protein-coding genes, miRNAs can also be regulated by Gli2. Our study raises the possibility that Gli2 functions as a global modifier of gene expression through the regulation of miRNA transcription. However, it is not known whether there are other miRNAs that might be directly modulated by Gli2. Further investigations will provide insight into how great a portion of the pri-miRNAs are regulated by Gli2 to fully understand the regulatory mechanism of Gli2 and miRNAs.

The biogenesis of miRNAs in mammalian systems is composed of multiple steps, including transcription of primary miRNA (pri-miRNA), cleavage of pri-miRNA to precursor miRNA (pre-miRNA), nucleocytoplasmic transport of pre-miRNA and cleavage of pre-miRNA to an miRNA duplex (31). Our findings suggested that miR-124 is transcriptionally regulated by Gli2 through binding of the upstream region of the miR-124 transcription start site, which contains a putative Gli2-binding element. This Gli2-mediated transcriptional regulation of miR-124 is mediated through direct binding of Gli2 to the upstream region of the transcriptional start site for miR-124. These findings suggest that transcription-dependent modulation of miRNA-124 biogenesis is governed by Gli2. Apart from the important role of Gli2 as a sequence-specific transcription factor, whether there is a transcription-independent mechanism is unclear.

Consistent with Gli2 function, many signature miRNAs, especially miR-124, are considered tumor-associated molecules, and miR-124 expression is lost in diverse types of tumors (32-34). miR-124 has previously been reported to be downregulated in glioma. A significant difference was found between glioma patients with a low miR-124 expression level, who had distinctly shorter survival times, and patients with a high miR-124 expression level (32). miR-124 usually regulates its target genes at the post-transcriptional level, and it is involved in multiple biological processes, including proliferation and metastasis. In renal clear cell carcinoma, miR-124 targets CAV1 and FLOT1 to inhibit cell proliferation (35). Through bioinformatic analysis and luciferase assays, we discovered that miR-124 interacts with the 3'-UTR region of AURKA (serine/threonine kinase, aurora kinase A). Moreover, AURKA was downregulated by miR-124 overexpression and upregulated by miR-124 knockdown.

AURKA, also referred to as Aurora-2, BTAK, ARK1, and STK15, maintains cell division by regulating centrosome separation, bipolar spindle assembly, and chromosome segregation (36). AURKA dysfunction can cause aneuploidy, mitotic arrest, genetic instability, poor histologic differentiation, and poor prognosis in various types of cancers, including colorectal, pancreatic, gastric, and breast cancers (37). AURKA expression can transform cells and drive tumor formation in mice (38). In addition, AURKA can block p53 function, thereby preventing cell apoptosis (39). Finally, AURKA has been shown to cooperate with RAS to induce malignant transformation (40). AURKA is a target of several miRNAs in various cancers. In non-small cell lung cancer, miR-32 can suppress NSCLC by targeting AURKA (41). Furthermore, increased expression of miR-25 downregulates the expression of the E3 ubiquitin ligase FBXW7, resulting in elevated levels of AURKA (42). In this study, we identified miR-124 as a direct negative regulator of AURKA. miR-124 directly repressed the expression of AURKA mRNA and protein through binding to one binding site in the 3'-UTR of the human AURKA gene, thereby negatively regulating AURKA functions. We have determined that miR-124 influences glioma cell proliferation by targeting AURKA.

In summary, our data indicate that aberrant expression of miR-124 through Gli2 inhibition in glioma cells can lead to the repression of AURKA, which can repress cell proliferation in glioma cells. Our results highlight an additional mechanism by which the Hh signaling pathway controls gene expression and influences cancer progression, and they elucidate a new mechanism through which the Hh signaling pathway regulates glioma development.

\section{Acknowledgements}

This work was supported in part by grants from the National Natural Science Foundation of China (31560314 to Q.L.) and the Natural Science Foundation of Jiangxi Province (2016BAB204168 to Q.L.).

\section{References}

1. Sul J and Fine HA: Malignant gliomas: New translational therapies. Mt Sinai J Med 77: 655-666, 2010.

2. Martín V, Herrera F, Carrera-Gonzalez P, García-Santos G, Antolín I, Rodriguez-Blanco J and Rodriguez C: Intracellular signaling pathways involved in the cell growth inhibition of glioma cells by melatonin. Cancer Res 66: 1081-1088, 2006.

3. Jiang $\mathbf{J}$ and Hui CC: Hedgehog signaling in development and cancer. Dev Cell 15: 801-812, 2008.

4. Ruiz i Altaba A: Gli proteins and Hedgehog signaling: Development and cancer. Trends Genet 15: 418-425, 1999.

5. Hui CC and Angers S: Gli proteins in development and disease. Annu Rev Cell Dev Biol 27: 513-537, 2011.

6. Bai CB, Auerbach W, Lee JS, Stephen D and Joyner AL: Gli2, but not Gli1, is required for initial SHh signaling and ectopic activation of the SHh pathway. Development 129: 4753-4761, 2002.

7. Mo R, Freer AM, Zinyk DL, Crackower MA, Michaud J, Heng HH, Chik KW, Shi XM, Tsui LC, Cheng SH, et al: Specific and redundant functions of Gli2 and Gli3 zinc finger genes in skeletal patterning and development. Development 124: 113-123, 1997.

8. Brechbiel J, Miller-Moslin K and Adjei AA: Crosstalk between hedgehog and other signaling pathways as a basis for combination therapies in cancer. Cancer Treat Rev 40: 750-759, 2014.

9. Santoni M, Burattini L, Nabissi M, Morelli MB, Berardi R, Santoni G and Cascinu S: Essential role of Gli proteins in glioblastoma multiforme. Curr Protein Pept Sci 14: 133-140, 2013.

10. Karhadkar SS, Bova GS, Abdallah N, Dhara S, Gardner D, Maitra A, Isaacs JT, Berman DM and Beachy PA: Hedgehog signalling in prostate regeneration, neoplasia and metastasis. Nature 431: 707-712, 2004.

11. Lee EY, Ji H, Ouyang Z, Zhou B, Ma W, Vokes SA, McMahon AP, Wong WH and Scott MP: Hedgehog pathway-regulated gene networks in cerebellum development and tumorigenesis. Proc Natl Acad Sci USA 107: 9736-9741, 2010. 
12. Ferruzzi P, Mennillo F, De Rosa A, Giordano C, Rossi M, Benedetti G, Magrini R, Pericot Mohr G, Miragliotta V, Magnoni L, et al: In vitro and in vivo characterization of a novel Hedgehog signaling antagonist in human glioblastoma cell lines. Int J Cancer 131: E33-E44, 2012.

13. Von Hoff DD, LoRusso PM, Rudin CM, Reddy JC, Yauch RL, Tibes R, Weiss GJ, Borad MJ, Hann CL, Brahmer JR, et al: Inhibition of the hedgehog pathway in advanced basal-cell carcinoma. N Engl J Med 361: 1164-1172, 2009.

14. Lauth M, Bergström A, Shimokawa T and Toftgård R: Inhibition of GLI-mediated transcription and tumor cell growth by smallmolecule antagonists. Proc Natl Acad Sci USA 104: 8455-8460, 2007.

15. Varnat F, Duquet A, Malerba M, Zbinden M, Mas C, Gervaz P and Ruiz i Altaba A: Human colon cancer epithelial cells harbour active HEDGEHOG-GLI signalling that is essential for tumour growth, recurrence, metastasis and stem cell survival and expansion. EMBO Mol Med 1: 338-351, 2009.

16. Adams BD, Kasinski AL and Slack FJ: Aberrant regulation and function of microRNAs in cancer. Curr Biol 24: R762-R776, 2014.

17. Lewis BP, Burge CB and Bartel DP: Conserved seed pairing, often flanked by adenosines, indicates that thousands of human genes are microRNA targets. Cell 120: 15-20, 2005.

18. Croce CM: Causes and consequences of microRNA dysregulation in cancer. Nat Rev Genet 10: 704-714, 2009.

19. Yan R, Peng X, Yuan X, Huang D, Chen J, Lu Q, Lv N and Luo S: Suppression of growth and migration by blocking the Hedgehog signaling pathway in gastric cancer cells. Cell Oncol (Dordr) 36: 421-435, 2013

20. Ciafrè SA, Galardi S, Mangiola A, Ferracin M, Liu CG, Sabatino G, Negrini M, Maira G, Croce CM and Farace MG: Extensive modulation of a set of microRNAs in primary glioblastoma. Biochem Biophys Res Commun 334: 1351-1358, 2005.

21. Silber J, Lim DA, Petritsch C, Persson AI, Maunakea AK, Yu M, Vandenberg SR, Ginzinger DG, James CD, Costello JF, et al: miR-124 and miR-137 inhibit proliferation of glioblastoma multiforme cells and induce differentiation of brain tumor stem cells. BMC Med 6: 14, 2008.

22. Xia H, Cheung WK, Ng SS, Jiang X, Jiang S, Sze J, Leung GK, Lu G, Chan DT, Bian XW, et al: Loss of brain-enriched miR-124 microRNA enhances stem-like traits and invasiveness of glioma cells. J Biol Chem 287: 9962-9971, 2012.

23. Goldenson B and Crispino JD: The aurora kinases in cell cycle and leukemia. Oncogene 34: 537-545, 2015

24. Tang X, Deng L, Chen Q, Wang Y, Xu R, Shi C, Shao J, Hu G, Gao M, Rao H, et al: Inhibition of Hedgehog signaling pathway impedes cancer cell proliferation by promotion of autophagy. Eur J Cell Biol 94: 223-233, 2015.

25. Raver-Shapira N, Marciano E, Meiri E, Spector Y, Rosenfeld N, Moskovits N, Bentwich Z and Oren M: Transcriptional activation of miR-34a contributes to p53-mediated apoptosis. Mol Cell 26: 731-743, 2007.

26. O'Donnell KA, Wentzel EA, Zeller KI, Dang CV and Mendell JT: c-Myc-regulated microRNAs modulate E2F1 expression. Nature 435: 839-843, 2005.

27. Ferretti E, De Smaele E, Miele E, Laneve P, Po A, Pelloni M, Paganelli A, Di Marcotullio L, Caffarelli E, Screpanti I, et al: Concerted microRNA control of Hedgehog signalling in cerebellar neuronal progenitor and tumour cells. EMBO J 27 2616-2627, 2008.
28. Hyun J, Wang S, Kim J, Rao KM, Park SY, Chung I, Ha CS, Kim SW, Yun YH and Jung Y: MicroRNA-378 limits activation of hepatic stellate cells and liver fibrosis by suppressing Gli3 expression. Nat Commun 7: 10993, 2016.

29. Yu F, Zheng Y, Hong W, Chen B, Dong P and Zheng J: MicroRNA-200a suppresses epithelial-to-mesenchymal transition in rat hepatic stellate cells via GLI family zinc finger 2. Mol Med Rep 12: 8121-8128, 2015.

30. Jiang Z, Cushing L, Ai X and Lü J: miR-326 is downstream of Sonic hedgehog signaling and regulates the expression of Gli2 and smoothened. Am J Respir Cell Mol Biol 51: 273-283, 2014.

31. Winter J, Jung S, Keller S, Gregory RI and Diederichs S: Many roads to maturity: microRNA biogenesis pathways and their regulation. Nat Cell Biol 11: 228-234, 2009.

32. Chen T, Wang XY, Li C and Xu SJ: Downregulation of microRNA-124 predicts poor prognosis in glioma patients. Neurol Sci 36: 131-135, 2015.

33. Hu CB, Li QL, Hu JF, Zhang Q, Xie JP and Deng L: miR-124 inhibits growth and invasion of gastric cancer by targeting ROCK1. Asian Pac J Cancer Prev 15: 6543-6546, 2014.

34. Peng XH, Huang HR, Lu J, Liu X, Zhao FP, Zhang B, Lin SX, Wang L, Chen HH, Xu X, et al: MiR-124 suppresses tumor growth and metastasis by targeting Foxq1 in nasopharyngeal carcinoma. Mol Cancer 13: 186, 2014.

35. Butz H, Szabó PM, Khella HW, Nofech-Mozes R, Patocs A and Yousef GM: miRNA-target network reveals miR-124as a key miRNA contributing to clear cell renal cell carcinoma aggressive behaviour by targeting CAV1 and FLOT1. Oncotarget 6: 12543-12557, 2015.

36. Glover DM, Leibowitz MH, McLean DA and Parry H: Mutations in aurora prevent centrosome separation leading to the formation of monopolar spindles. Cell 81: 95-105, 1995.

37. Gautschi O, Heighway J, Mack PC, Purnell PR, Lara PN Jr and Gandara DR: Aurora kinases as anticancer drug targets. Clin Cancer Res 14: 1639-1648, 2008.

38. Bischoff JR, Anderson L, Zhu Y, Mossie K, Ng L, Souza B, Schryver B, Flanagan P, Clairvoyant F, Ginther C, et al: A homologue of Drosophila aurora kinase is oncogenic and amplified in human colorectal cancers. EMBO J 17: 3052-3065, 1998.

39. Katayama H, Sasai K, Kawai H, Yuan ZM, Bondaruk J, Suzuki F, Fujii S, Arlinghaus RB, Czerniak BA and Sen S: Phosphorylation by aurora kinase A induces Mdm2-mediated destabilization and inhibition of p53. Nat Genet 36: 55-62, 2004.

40. Tatsuka M, Sato S, Kitajima S, Suto S, Kawai H, Miyauchi M, Ogawa I, Maeda M, Ota T and Takata T: Overexpression of Aurora-A potentiates HRAS-mediated oncogenic transformation and is implicated in oral carcinogenesis. Oncogene 24: 1122-1127, 2005

41. Ma ZL, Zhang BJ, Wang DT, Li X, Wei JL, Zhao BT, Jin Y, Li YL and Jin YX: Tanshinones suppress AURKA through up-regulation of miR-32 expression in non-small cell lung cancer. Oncotarget 6: 20111-20120,2015.

42. Li Z, Sun Y, Chen X, Squires J, Nowroozizadeh B, Liang C and Huang J: p53 mutation directs AURKA overexpression via miR-25 and FBXW7 in prostatic small cell neuroendocrine carcinoma. Mol Cancer Res 13: 584-591, 2015. 